\title{
OPEN SIMPLE MAPS AND PERIODIC HOMEOMORPHISMS ${ }^{1}$
}

\author{
JOHN D. BAILDON
}

\begin{abstract}
It is shown that an open map between 2-manifolds without boundary that is the composition of $n$ open simple (at most two-to-one) maps is necessarily of order $2^{n}$. Since $w=z^{3}$ on the unit sphere is of order three, this shows an open map cannot necessarily be factored into a composition of open simple ones.
\end{abstract}

1. Introduction. In [1] K. Borsuk and R. Molski studied a class of mappings, called the mappings of finite order. A mapping defined on a space $X$ is said to be of order $\leqq k$ if for any' $y$ in $f(X), f^{-1}(y)$ contains at most $k$ points. They take particular interest in the mappings of order $\leqq 2$, which are called simple maps. We shall also require that a simple map have at least one point inverse with cardinality two. Borsuk and Molski provide a number of examples of simple maps and pose several problems concerning them. Among these is the question of whether or not there exists a continuous mapping of finite order which is not a superposition (composition) of a finite number of simple mappings. In [3] K. Sieklucki shows that every such map on a compact, finite dimensional space is such a superposition and gives an example of a map of finite order on an infinite dimensional compact space which cannot be so reduced. J. W. Jaworowski points out in [2] that an open simple map on a compact space induces a continuous involution (and hence a homeomorphism) on the space and vice versa. Thus there is a direct connection between the study of open simple maps and that of homeomorphisms of period two.

By the singular set $B_{f}$ of a map $f$ from a space $X$ onto $Y$, we will mean the set of all points in $X$ where $f$ is not locally one-to-one. In the case of an open simple map on a compact $n$-manifold, $B_{f}$ consists of the set of singleton point inverses.

Presented to the Society, June 28, 1971; received by the editors October 20,1971 and, in revised form, September 15, 1972.

AMS (MOS) subject classifications (1970). Primary 54C10; Secondary 54B15, 54H15.

Key words and phrases. Simple map, finite-to-one map, periodic homeomorphism, superposition of maps, composition of maps, singular sets, fixed point sets, manifolds.

${ }^{1}$ This paper is a portion of the author's Ph.D. thesis written under the direction of Professor Louis F. McAuley at the State University of New York at Binghamton. The author would like to express his gratitude to the referee for his many helpful suggestions.

(c) American Mathematical Society 1973 
2. Compositions of open simple maps. In [1] Borsuk and Molski defined a map to be elementary if its domain is metric and if there exists a positive number $\varepsilon$ so that for every two different points $x$ and $x^{\prime}$, if $f(x)=f\left(x^{\prime}\right)$, then $d\left(x, x^{\prime}\right) \geqq \varepsilon$. They then show that every elementary mapping over a compactum is a superposition (composition) of a finite number of simple mappings. They also raise the

Question. Does there exist a continuous mapping of finite order which is not a superposition of a finite number of simple mappings?

Sieklucki has answered this in part with the following [3]:

THEOREM 1 (SIEKLUCKI). Every continuous mapping $f$ of order $\leqq k$ $(k \geqq 2)$ defined on a compact $n$-dimensional space $X$ is a finite superposition of simple mappings.

He also gives an example of a continuous map of finite order defined on a compact infinite dimensional space which is not a finite superposition of simple maps. It thus seems natural to ask:

Question. Is every open mapping $f$ of order $\leqq k(k \geqq 2)$ defined on a compact $n$-dimensional space necessarily a finite superposition of open simple maps?

That this is not the case is demonstrated by the following:

THEOREM 2. If the open map f between 2-manifolds without boundary is the superposition of $n$ open simple maps, then $f$ is of order $2^{n}$.

Proof. Suppose $f=f_{n} \circ f_{n-1} \circ \cdots \circ f_{1}$, where $f_{i}$ is an open simple map from $A_{i-1}$ onto $A_{i}$ with $A_{0}=A$ and $A_{n}=B$. Whyburn has shown that the $A_{i}$ will all be 2-manifolds [4, X, 4.4]. Since $B$ has no boundary and the $f_{i}$ 's are open, the $A_{i}$ 's must also have no boundary. Whyburn has also shown that $f_{i}\left(B_{f_{i}}\right)$ is finite $[4, \mathrm{X}, 6.3]$ so that all but a finite number of the point inverses of $f_{i}$ contain exactly two points. The proof proceeds by induction assuming that any map between 2-manifolds without boundary which is the superposition of $n-1$ open simple maps has the property that all but a finite number of point inverses contain exactly $2^{n-1}$ points. The map $f$ will then have its point inverses containing $2^{n}$ points except for the (finite number of) points in $B=A_{n}$ where $f_{n}$ has singleton point inverses or the points (finite in number by the induction assumption) which are images of points where $f_{n-1} \circ \cdots \circ f_{1}$ has point inverses which are not of cardinality $2^{n-1}$. Thus the cardinality of $f^{-1} f(y)$ is $2^{n}$ except at a finite number of $y$ in $B$ and so $f$ is $2^{n}$-to-one.

Since a three-to-one map such as $w=z^{3}$ on the unit sphere could not consequently be a finite superposition of open simple maps, we have obtained a negative answer to our question. 
Nevertheless, such factorings are sometimes possible, in which case we will make use of the following theorem by Whyburn to obtain some information about the map $[4, X, 7.3]$ :

THEOREM 3 (WhyBuRN). If $A$ and $B$ are compact 2-dimensional manifolds without boundary and $f(A)=B$ is a light open map of degree $k$ (i.e., is $k$-to-one), then $k \chi(B)-\chi(A)=k r-n$, where $r$ and $n$ are the cardinalities of $Y$ and $f^{-1}(Y)$, respectively, when $Y$ is the set of all $y$ in $B$ such that $f^{-1}(y)$ contains a point of $A$ at which $f$ is not locally topological (i.e., $Y$ is the image of the singular set of $f$ ) and $\chi(Z)$ represents the Euler characteristic of $Z$.

THEOREM 4. If a k-to-one map between compact 2-manifolds without boundary, $A$ and $B$, can be factored into open simple maps $f_{1}, \cdots, f_{s}$, where $f_{i}$ maps $A_{i-1}$ onto $A_{i}$ with $A_{0}=A$ and $A_{s}=B$, then $k r-n=\sum_{i=1}^{s} 2^{i-1} r_{i}$ where $r_{i}$ is the number of singular points of $f_{i}, r$ is the number of points in the image of the singular set, $f\left(B_{f}\right)$, and $n$ is the cardinality of $f^{-1} f\left(B_{f}\right)$.

Proof. As in Theorem 1, the $A_{i}$ will all be 2-manifolds without boundary. Applying Theorem 2 to the maps $f_{i}$, we get $2 \chi\left(A_{i}\right)-\chi\left(A_{i-1}\right)=2 r_{i}-n_{i}$ where $n_{i}$ is the cardinality of $f_{i}^{-1} f_{i}\left(B_{f_{i}}\right)$. Since $B_{f_{i}}$ is the set of all singleton point inverses, $n_{i}=r_{i}$ and so $2 \chi\left(A_{i}\right)-\chi\left(A_{i-1}\right)=r_{i}$. Theorem 2 also gives us $k r-n=k \chi(B)-\chi(A)=2^{s} \chi\left(A_{s}\right)-\chi\left(A_{0}\right)$. Thus we can prove our theorem by showing that $\chi\left(A_{0}\right)=2^{s} \chi\left(A_{s}\right)-\sum_{i=1}^{s} 2^{i-1} r_{i}$. If $s=1$ this is true by our earlier remark on $f_{i}$. Proceeding inductively, we assume that $\chi\left(A_{0}\right)=$ $2^{s-1} \chi\left(A_{s-1}\right)-\sum_{i=1}^{s-1} 2^{i-1} r_{i}$. The result follows from the fact that $2 \chi\left(A_{s}\right)-$ $\chi\left(A_{s-1}\right)=r_{s}$.

3. Periodic homeomorphisms and compositions. As we mentioned earlier, Jaworowski has shown that an open simple map on a compact space induces an involution and vice versa. Thus there is a direct connection between the study of open simple maps and that of homeomorphisms of period 2. Since the singular set of an open simple map on a compact set is the set of singleton point inverses, it corresponds to the fixed point set of the induced involution. The corollary to the next result indicates there is also a relationship between homeomorphisms of period $2^{k}$ and maps which can be factored into open simple ones. The author wishes to thank the referee for his assistance in the following proof.

THEOREM 5. Let $h$ be a periodic homeomorphism of period $p_{1} p_{2} \cdots p_{k}$ defined on a space $X$. Then the orbit map f associated with $h$ can be written as the superposition of $k$ open maps of orders $p_{1}, p_{2}, \cdots, p_{k}$.

Proof. In general if a group $G$ acts on a space $X$ and $H$ is a normal subgroup of $G$, then the quotient group $G / H$ acts on the orbit space $X / H$ in a natural way and induces the bijection $g$ from $X / G$ to $(X / H) /(G / H)$. 
Examination of the natural diagram involving $X, X / H, X / G$, and $(X / H) /(G / H)$ shows that $g$ is a homeomorphism. Hence the orbit map from $X$ to $X / G$ factors into the two orbit maps from $X$ to $X / H$ and from $X / H$ to $X / G$, both of which are open. A homeomorphism of period $p_{1} \cdots p_{k}$ is an action of $Z_{p_{1} \cdots p_{k}}$ and so the factorization $X \rightarrow X\left|Z_{p_{k}} \rightarrow X\right| Z_{p_{k-1} p_{k}} \rightarrow \cdots \rightarrow$ $X / Z_{p_{1} \cdots p_{k}}$ produces the result we seek.

In particular, if $h$ is of order $2^{k}$, we have

COROLlary 6. Let $h$ be a homeomorphism of period $2^{k}$ defined on a space $X$. Then the orbit map $f$ associated with $h$ can be written as the superposition of $k$ open simple maps.

\section{REFERENCES}

1. K. A. Borsuk and R. Molski, On a class of continuous mappings, Fund. Math. 45 (1957), 84-98. MR 21 \#858.

2. J. W. Jaworowski, On simple regular mappings, Fund. Math. 45 (1958), 119-129. MR 21 \#859.

3. K. Sieklucki, On superpositions of simple mappings, Fund. Math. 48 (1960), 217228. MR 22 \#2981.

4. G. T. Whyburn, Analytic topology, Amer. Math. Soc. Colloq. Publ., vol. 28, Amer. Math. Soc., Providence, R.I., 1963. MR 32 \#425.

Department of Mathematics, Pennsyluania State University, Worthington Scranton Campus, Dunmore, Pennsylvania 18512 